\title{
Avaliação ecocardiográfica do desenvolvimento cardíaco de cavalos atletas: comparação entre atividades físicas distintas ${ }^{1}$
}

\author{
Carolina C.M. Bonomo ${ }^{2 *}$, Lilian E.S. Michima², Patricia Miyashiro ${ }^{2}$ \\ e Wilson Roberto Fernandes ${ }^{2}$
}

\begin{abstract}
Bonomo C.C.M., Michima L.E.S., Miyashiro P. \& Fernandes W.R. 2014 [Echocardiographic evaluation of cardiac development of athletic horses: comparision between different physical activities.] Avaliação ecocardiográfica do desenvolvimento cardíaco de cavalos atletas: comparação entre atividades físicas distintas. Pesquisa Veterinária Brasileira 34(9):923-927. Departamento de Clínica Médica,, Faculdade de Medicina Veterinária e Zootecnia, Universidade de São Paulo, Av. Orlando Marques de Paiva 87, São Paulo, SP 05508-270, Brazil. E-mail: carolinabonomo@yahoo.com.br

The aerobic capacity of equine athletes may be explained, in part, by its cardiac development associated with physical training. Echocardiographic examination of equine athletes allows the visualization of these changes and cardiac function of these animals. We aimed to evaluate echocardiographically horses of different sports to see if the type of physical activity performed by each group of animals promotes structural or functional cardiac differences. 196 horses underwent echocardiography at rest were divided into three groups: Polo group, 44 horses participating in tests of Polo; QM group, 49 horses of Quarter Horse, participants of barrel and pole bending events and PSI group, 103 horses of the breed Thoroughbred racehorses. We evaluated the following heart rates: IVS, LVD and LVFW in systole and diastole, LA in systole, Ao in diastole, mean LA: Ao, EF, EV, LVFSV, LVFDV, FS\%, E-S, LVET, CO and HR. The PSI group animals showed higher left ventricular volumes and provided proportionately less efficiency when we analyzed EV. Animals in group Polo and QM showed higher values when variables such as cardiac function. The more intense physical activity performed by the animals of group QM and Polo provided better functional development of the heart of these animals, and the PSI group, presented figures suggesting that aerobic capacity can still be better exploited through better targeting of their physical training.
\end{abstract}

INDEX TERMS: Equine, echocardiography, Polo, Quarter horse, Thoroughbred.

RESUMO.- A capacidade aeróbica dos equinos atletas pode ser explicada, em partes, pelo seu desenvolvimento cardíaco associado ao treinamento físico. 0 exame ecocardiográfico de equinos atletas permite a visualização destas alterações e da função cardíaca destes animais. Buscou-se avaliar ecocardiograficamente equinos de diferentes modalidades esportivas, para verificar se o tipo de atividade física desempenhada por cada grupo de animais promove diferenças estruturais ou funcionais cardíacas. 196 equinos

\footnotetext{
${ }^{1}$ Recebido em 8 de janeiro de 2014.

Aceito para publicação em 27 de julho de 2014

${ }^{2}$ Departamento de Clínica Médica, Faculdade de Medicina Veterinária e Zootecnia (FMVZ), Universidade de São Paulo (USP), Av. Orlando Marques de Paiva 87, São Paulo, SP 05508-270, Brasil. *Autor para correspondência: carolinabonomo@yahoo.com.br
}

foram submetidos à avaliação ecocardiográfica em repouso, sendo divididos em três grupos: grupo Polo, 44 equinos participantes de provas de Polo; grupo QM, 49 equinos da raça Quarto de Milha, participantes de provas de baliza e tambor, e grupo PSI, 103 equinos da raça Puro Sangue Inglês participantes de provas de corridas. Foram avaliados os seguintes índices cardíacos: SIV, DIVE e PLVE em sístole e diástole, $\mathrm{AE}$ em sístole, Ao em diástole, relação $\mathrm{AE}: \mathrm{Ao}, \mathrm{FEj}$, VEj, VSFVE, VDFVE, FS\%, E-S, TEVE, DC e FC. Os animais do grupo PSI apresentaram os maiores volumes de ventrículo esquerdo, tendo apresentado proporcionalmente menor eficiência quando analisado o VEj. Os animais do grupo Polo e QM apresentaram melhores valores quando analisadas as variáveis de função cardíaca. A atividade física mais intensa desempenhada pelos animais do grupo 
QM e Polo permitiu melhor desenvolvimento funcional do coração destes animais, tendo o grupo PSI, apresentado valores que sugerem que sua capacidade aeróbica ainda pode ser melhor explorada através de melhor orientação de seu treinamento físico.

TERMOS DE INDEXAÇÃO: Equinos, ecocardiografia, Polo, Quarto de Milha, Puro Sangue Inglês.

\section{INTRODUÇÃo}

0 treinamento físico aeróbio exerce a papel notável sobre o sistema cardiorrespiratório, mais do que qualquer um dos vários estresses vivenciados na atividade cotidiana. 0 estudo da fisiologia do exercício representa um método importante para melhorar a compreensão de como o sistema cardiovascular e respiratório respondem e interagem nesta situação (Laughlin 1999). A maioria dos efeitos cardiorrespiratórios decorrentes do exercício está relacionada ao suprimento adequado de oxigênio e nutrientes para os músculos em trabalho (Laughlin 1999).

Particularmente, o sistema cardiovascular dos equinos apresenta função e características que permitem que estes animais atinjam grande desempenho atlético. Durante a atividade física, a alta capacidade cardíaca é altamente requisitada, e ainda assim, há um desbalanço entre a resposta cardíaca e a capacidade pulmonar, visto que a seleção destes animais têm, evidentemente, gerando aumento no tamanho cardíaco e capacidade do mesmo em bombear o sangue, porém a capacidade pulmonar não parece acompanhar na mesma proporção - a consequência disto é a insuficiência do sistema respiratório durante o exercício máximo (Lyons 1987).

Entre as alterações cardíacas mais comumente associadas ao treinamento físico temos o aumento do tamanho do ventrículo esquerdo, aumento da massa do ventrículo esquerdo e espessamento de sua parede. Essas alterações são responsáveis pelo chamado "coração de atleta" (Epllicia 1997). De acordo com Morganroth et al. (1975), podemos encontrar dois tipo de coração de atleta: aquele que é submetido a treinamento de força e o que acontece após o treinamento de resistência. Atletas envolvidos com atividades esportivas com alto componente dinâmico, como por exemplo corrida, desenvolvem predominantemente aumento da câmara cardíaca esquerda com aumento proporcional da parede causado pelo aumento de carga de volume associado ao alto débito cardíaco, ou seja um hipertrofia excêntrica do ventrículo esquerdo; enquanto que atletas envolvidos com atividade física isométrica, como levantamento de peso, apresentam predominantemente aumento da espessura da parede ventricular sem alteração do tamanho do ventrículo esquerdo, caracterizando este processo por um hipertrofia concêntrica.

Entre as diferentes raças de equinos, os cavalos Puro Sangue Inglês, que participam de provas de corrida, apresentam os maiores tamanhos de coração, e, de acordo com Evans \& Rose (1988), há uma relação linear entre o peso do coração destes animais e o débito cardíaco, e entre o débito cardíaco e o consumo de oxigênio, permitindo que estes animais, que apresentem corações saudáveis e maiores, cheguem a 400-500 L/min de débito cardíaco e um consumo de oxigênio de $240 \mathrm{ml} \mathrm{02} / \mathrm{kg} / \mathrm{min}$.

0 exame ecocardiográfico representa um método diagnóstico amplamente difundido, com um poderoso argumento de aproximar o conhecimento teórico de sua aplicação prática. A imagem ultrassonográfica pode gerar imagens das estruturas cardíacas e demonstrar como estas se movimentam em certo período de tempo, oferecendo a facilidade de acessar parâmetros relacionados à função cardíaca (Brunner 1995). Além disso, este tipo de técnica não é invasiva, não causa dor ao paciente, e não apresenta efeitos colaterais. Deve-se ressaltar também, que as medidas podem ser repetidas o quanto necessário, sem que tragam qualquer prejuízo ao animal (Brunner 1995).

Técnicas ecocardiográficas têm demonstrado haver uma significante relação entre a massa do ventrículo esquerdo e o consumo de oxigênio (Young 2002) Além disso, relações entre o tamanho do ventrículo esquerdo, função sistólica e desempenho em corridas estão crescendo entre as pesquisas na área (Young 2005). Estes achados oferecem alguma perspectiva de métodos de avaliação não invasivos que podem ter valor ao ajudar a selecionar o potencial atlético de um animal (Poole \& Erickson 2008).

No presente trabalho, buscou-se a avaliação ecocardiográfica de equinos que desempenham diferentes tipos de atividade física para verificar se o coração dos equinos que praticam diferentes esportes apresenta diferença significativa, e qual influência da raça nestes padrões dos animais em repouso.

\section{MATERIAL E MÉTODOS}

Foram avaliados 196 equinos atletas, de qualquer idade, sexo e peso, divididos em 3 grupos, sendo:

Grupo Polo: 44 equinos participantes de provas de polo (sendo estes mestiços de Quarto de Milha e Puro Sangue Inglês);

Grupo QM: 49 equinos da raça Quarto de Milha, participantes de provas de tambor e/ou baliza;

Grupo PSI: 103 equinos da raça Puros Sangue Inglês, participantes de provas de corrida.

Todos os animais foram submetidos a exame físico prévio (frequência cardíaca, frequência respiratória, temperatura retal, movimentos cecais/3 minutos e caracterização do pulso arterial), seguido de exame específico do sistema cardiovascular (coloração de mucosas, tempo de preenchimento capilar, preenchimento da veia jugular, auscultação de todos os focos cardíacos ambos hemitórax, avaliação do choque pré-cordial e grau de hidratação). Animais que apresentaram alteração em quaisquer uns dos parâmetros avaliados e com histórico de doença anterior (incluindo HYPP para os animais Quarto de Milha) foram excluídos do estudo. Além disso, registrou-se histórico de todos animais contendo as seguintes informações: sexo, idade, pelagem, peso corpóreo, regime de criação, dieta, atividade desempenhada, esquema de treinamento.

Os animais foram avaliados em repouso somente. Para o exame ecocardiográfico, os animais encontravam-se calmos, contidos apenas pelo cabresto, em um ambiente tranquilo e de pouca luz, como sugerido por Vöros (1991). Seguiu-se com a limpeza da região, compreendida entre o 3 o e 5o espaços intercostais da janela cardíaca direita, acima do olécrano e caudal ao músculo tríceps, 
com água e sabão, e se aplicou quantidade suficiente de gel de contato ultrassonográfico para uma visualização adequada das estruturas cardíacas, evitando a presença de ar entre pêlo e transdutor (LONG, 1992; REEF, 1998).

Por questões estéticas, considerando que os animais estavam em fase de competição, não foi feita a tricotomia da região para realização do exame, assim, foi aplicada uma pequena quantidade de gel de contato ultrassonográfico a mais no local para diminuir a presença de ar entre pelo e transdutor, antes da aplicação do gel de contato ultrassonográfico no transdutor.

Para facilitar o exame na janela cardíaca direita, o membro anterior direito encontrava-se posicionado um pouco à frente do membro contralateral, como sugerido por Long (1992) e Reef (1998).

O aparelho de ultrassom utilizado foi o 180 Plus versão 1.9, Sonosite $\AA$, com capacidade de realizar exames no modo-M (unidimensional), modo B (bidimensional) e Doppler, equipado com transdutor C15 Sonosite $®$, microconvexo, eletrônico e multi-setorial de 2 a 4 megahertz (MHz). 0 transdutor foi ajustado a uma profundidade de $25 \mathrm{~cm}$ e a uma frequência de $2 \mathrm{MHz}$.

Foram utilizados os modos B (bidimensional) e M (unidimensional) para a realização do exame ecocardiográfico. Em ambos, foi possível avaliar simetria de movimentação das estruturas, inexistências de alterações anatômicas ou que sugerissem presença de doença.

Ao exame do modo B foi mensurado o seguinte índice cardíaco: átrio esquerdo em sístole (AEs).

Ao exame do modo $M$ foram avaliados os seguintes índices cardíacos: espessuras de septo interventricular (SIV), parede livre do ventrículo esquerdo (PLVE) e diâmetro interno de ventrículo esquerdo (DVE), durante o fim da diástole (d) e pico de sístole (s); diâmetro aórtico em final de diástole (Ao), razão entre AE:Ao, distância do ponto E (maior abertura da valva mitral) ao septo interventricular (E-S), tempo de ejeção ventricular - da abertura ao fechamento dos folhetos da valva aórtica (TEVE), fração de encurtamento do ventrículo esquerdo (FS\%), fração de ejeção do ventrículo esquerdo (FEj), volume de ejeção do ventrículo esquerdo (VEj), volume sistólico final do ventrículo esquerdo (VSFVE), volume diastólico final do ventrículo esquerdo (VDFVE), débito cardíaco (DC) e frequência cardíaca (FC).

A técnica ecocardiográfica utilizada foi a técnica descrita por Reef (1998). Realizou-se a análise descritiva dos dados por meio de média, desvio padrão, medidas de posição (mínimo, máximo, mediana e quartis) para as variáveis quantitativas. Após isto, então, foi testada a diferença entre as médias dessas variáveis entre os grupos através do Teste t-student.

Para as variáveis categóricas (sexo, grau de hidratação, etc) verificou-se a distribuição de frequência absoluta e relativa e o Teste Qui-Quadrado para avaliar associação entre estas e os grupos.

Para avaliar de forma mais específica três variáveis de função escolhidas, sendo estas débito cardíaco (DC), fração de encurtamento (FS\%) e volume de ejeção (VEj), ajustou-se um modelo de regressão linear para estas, explicadas pelas variáveis estruturais e pelos grupos dos equinos estudados. Para avaliar quais medidas utilizar como variável explicativa, inicialmente, avaliou-se as correlações de Pearson .Aquelas que se apresentaram correlacionadas com a variável resposta foram inseridas num modelo de regressão que incluía o efeito dessas variáveis interagindo com a raça, e então aplicou-se um método stepwise AIC para seleção do modelo final. As suposições do modelo foram testadas através de gráficos QQ e de resíduos e o ajuste do modelo foi avaliado pela estatística $\mathrm{R}^{2}$.

Para a análise foi considerado um nível de significância de 5\% para a inferência nos testes. 2012.

\section{RESULTADOS}

Dentre o número total de animais estudados, a distribuição entre os grupos de acordo com o sexo, verificou-se predomínio significativo de animais do sexo feminino (75\%), não tendo os outros grupos apresentado diferença quanto a esta distribuição. Com relação ao peso, os animais que apresentaram maior peso foram os animais do grupo PSI $(470,9 \mathrm{~kg}$ $\pm 32,1)$, seguido pelos animais do grupo Polo $(457 \mathrm{~kg} \pm 35,6)$ e por último os animais do grupo QM $(442,9 \mathrm{~kg} \pm 34,8)$.

As médias de valores obtidos para as variáveis estruturais ecocardiográficas encontram-se descritos no Quadro 1.

Os valores obtidos para VSFVE e VDFVE, verificou-se notável aumento com relação ao grupo PSI ( $242,08 \mathrm{ml} \pm$ 76,10 e $711,04 \mathrm{ml} \pm 136,56$, respectivamente).

A correlação entre as variáveis de função cardíacas com alguns parâmetros selecionados podem ser melhor observados na Figura 1.

Quanto à realização de modelos de regressão linear, testamos a influência de todas as variáveis sobre as seguintes variáveis de função: débito cardíaco, volume de ejeção e fração de encurtamento. 0 grupo padrão para comparação foi o grupo Polo. Para a avaliação do DC, considerou-se como variáveis explicativas a Raça, o SIVs e o VDFVE. A comparação entre as Raças nos faz acreditar que os cavalos QM tem em média $1 \mathrm{l} / \mathrm{m}$ a menos de DC do que os cavalos PSI e Pólo. Independentemente da raça, estima-se que a média do DC cresça 2,316 unidades para o aumento de uma unidade de SIVs e cresça 1,8 unidades para o aumento de $100 \mathrm{ml}$ de VDFVE. 0 modelo propõe que a média do VEj dos cavalos da raça PSI sejam inferiores em $16 \mathrm{ml}$, em média, comparado às outras raças e que a variável VDFVE é a mais importante para explicar o VEj e estima-se que o aumento de $100 \mathrm{ml}$ do VDFVE produz um aumento médio de $57,5 \mathrm{ml}$ no volume de ejeção.

\section{Quadro 1. Valores médios obtidos através de exame ecocardiográfico modo-M}

\begin{tabular}{lcccc}
\hline Variável & Grupo & Média & Desvio padrão & Valor de $\mathrm{p}^{*}$ \\
\hline SIVd (cm) & Todos & 3,15 & 0,41 & $<0,001$ \\
Polo & 3,26 & 0,36 & & \\
PSI & 3,22 & 0,40 & & \\
QM & 2,89 & 0,37 & & \\
SIVs (cm) & Todos & 4,57 & 0,51 & $<0,001$ \\
Polo & 4,51 & 0,44 & & \\
PSI & 4,76 & 0,46 & & \\
QM & 4,20 & 0,45 & & \\
DVEd (cm) & Todos & 10,71 & 0,99 & $<0,001$ \\
Polo & 10,46 & 0,82 & & \\
PSI & 11,07 & 0,95 & & \\
QM & 10,18 & 0,93 & & \\
DVEs $(\mathrm{cm})$ & Todos & 6,50 & 0,94 & $<0,001$ \\
Polo & 6,19 & 0,91 & & \\
PSI & 6,79 & 0,92 & & \\
QM & 6,18 & 0,82 & & \\
PLVEd (cm) & Todos & 2,79 & 0,66 & \\
Polo & 2,76 & 0,63 & & \\
PSI & 2,57 & 0,37 & & \\
QM & 3,12 & 0,84 & & \\
PLVEs (cm) & Todos & 4,33 & 0,71 & \\
Polo & 4,41 & 0,71 & & \\
PSI & 4,17 & 0,51 & & \\
QM & 4,55 & 0,92 & & \\
& & & &
\end{tabular}




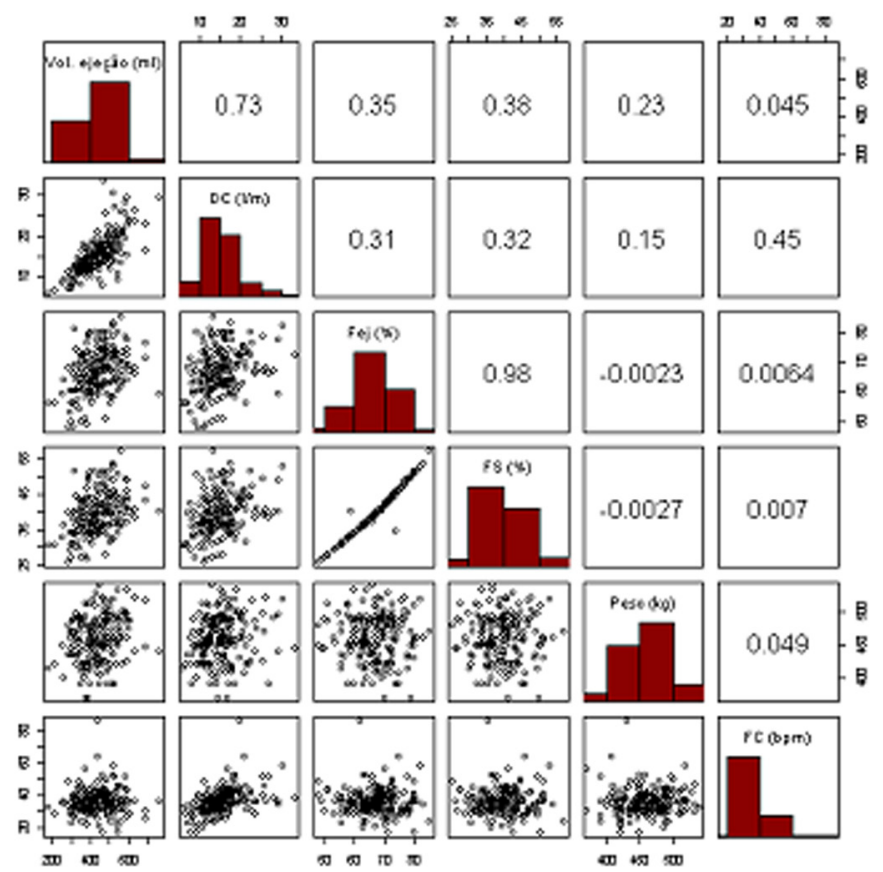

Fig.1. Correlação entre as variáveis cardíacas (verificar o valor de cada correlação através de linhas interseccionadas). Obs.: Quanto mais próximo a 1, maior a correlação entre as variáveis.

Com relação à análise da FS\% estima-se que o aumento de uma unidade de SIVs, acrescente, em média, 4,35\% a mais de FS\%. E o aumento de uma unidade de SIVd, decresça em 3,59\%, em média a FS\% dos cavalos. Além disso, o efeito de $100 \mathrm{ml}$ de VSFVE produz uma redução média de 4,9\% na variável resposta e os cavalos do grupo QM apresentam FS\% em média 2,1\% menores do que os outros grupos. O VSFVE é, aparentemente, o maior responsável pela redução da FS\%.

\section{DISCUSSÃO}

A técnica descrita para a realização do exame ecocardiográfico mostrou-se adequada durante a avaliação dos animais. Devido ao tamanho elevado das câmaras cardíacas dos cavalos do Grupo PSI, muitas vezes não foi possível mensurar a PLVE neste grupo, visto que a mesma encontrava-se fora da janela de alcance do aparelho de ultrassom utilizado. 0 fato de não ter sido realizada tricotomia, apesar de exigir quantidade excessiva de gel, não representou um obstáculo para a realização do exame, sem trazer prejuízos às imagens realizadas. Quando consideradas as referências anatômicas corretas para a realização do exame, não houve dificuldades para a identificação e mensuração das estruturas intra-cardíacas.

Assim como já citado por alguns autores, como Poole e Erickson (2008), o peso do coração parece estar relacionado com o peso corpóreo do animal, estando relacionado à sua hipertrofia (Figura 1). Ainda que esta possibilidade seja um consenso entre os estudos na área, neste trabalho, após análise de correlação, o peso não apresentou correlação significativa com nenhuma variável de função, sendo VEj, DC, e sendo até negativa sobre FEj e FS\%. Isto pode estar relacionado ao fato de que os animais mais pesados deste estudo não representaram, necessariamente, o melhor desempenho cardíaco, como é o caso do grupo PSI, que apresenta os animais mais pesados, estando provavelmente estes, aquém do seu desempenho.

Considerando que o SIV apresenta maior contribuição muscular durante a sístole, os animais dos grupos Polo e PSI apresentaram maior espessura do SIV, diferente do ocorrido com os animais do grupo QM. Devemos levar em conta, neste caso também, o fato de que nem todos os animais do grupo PSI tiveram a PLVE mensurada, como citado anteriormente, por uma dificuldade da técnica. Ainda assim, estes resultados surpreendem se considerarmos que os animais do grupo QM apresentam o maior componente de atividade de força, baseado no treinamento e provas que participam, e estes não apresentaram os maiores valores de espessura de suas paredes.

Os animais do grupo Polo apresentaram em geral, valores intermediários, principalmente ao analisarmos os valores de DVE, sendo possível esta observação por dois fatores: se considerarmos a atividade destes animais, que não tende ao metabolismo anaeróbio, como no caso do grupo QM, mas também, não possui características tão dinâmicas quanto os animais do grupo PSI; além disso, devemos lembrar que os animais deste grupo apresentam-se em sua maioria composta por animais mestiços, tendo em sua formação animais tanto da raça QM como PSI.

Quando analisado o grupo de QM, há tendência tanto de hipertrofia excêntrica como de concêntrica, bem evidente mais que nos outros grupos, visto que estes animais apresentaram os menores valores de DVE e os maiores de PLVE, sugerindo esta tendência. 0 que conflui com o fato, mais ou vez, destes animais desenvolverem atividade mais relacionada à força, ainda que sejam submetidos também a treinamento aeróbico, sua principal atividade remete à realização de exercício predominantemente anaeróbico. Esta característica de exercício dinâmico com componente anaeróbico, de acordo com Fagard (1997), deve levar ao aumento do DVE e aumento da espessura da PLVE, em atletas humanos, e a esta característica mista de hipertrofia cardíaca.

0 exercício deve promover aumento do relaxamento cardíaco, melhorando a função diastólica do coração de um atleta, permitindo assim o aumento do volume de ejeção, ou seja, o aumento do volume ejetado em um ciclo cardíaco aumenta em um atleta por haver aumento do volume diastólico (Laughlin 1999). Da mesma forma como já ocorreu com outras variáveis aqui analisadas, e sendo condizente com estas, os animais do grupo PSI apresentaram os maiores valores tanto de VDFVE como de VSFVE, seguidos pelos animais do grupo Polo e por último pelos animais do grupo QM. Curiosamente, no grupo PSI, o valor máximo encontrado foi de $1.300 \mathrm{ml}$, sendo condizente com o citado por Evans (1994), sugerindo que este animal deva apresentar grande desempenho em corridas (Young 2005).

Ao realizar a comparação entre FS\% e FEj, obteve-se o maior valor de correlação entre variáveis de função. Sendo assim, os animais com melhor FS\% apresentaram melhor FEj. Vale ressaltar que, neste trabalho, como não houve diferença entre os grupos quanto à $F E j$, a diferença de rendimento em questão de volume se deu principalmente pela 
diferença volumétrica do ventrículo esquerdo, que esta muito relacionada á característica racial, e sofrendo influência, não menos importante da atividade física.

Houve uma correlação muito maior $(0,73)$ do DC com VEj do que do DC com a FC $(0,45)$, sugerindo uma maior contribuição do VEj para os valores encontrados de débito cardíaco. Uma avaliação pós exercício pode trazer respostas sobre como estas variáveis atuam e interagem em uma situação de maior requerimento.

Para os grupos aqui estudados, pode-se observar que o DC sofreu influência dos grupos dos SIVs e do VDFVE. Dentro dos grupos, o grupo QM influenciou de forma negativa esta variável, já o grupo PSI não apresentou diferença se compararmos ao grupo Polo. Além disso, temos que o SIVs contribuiu de forma positiva com o DC, de forma que um aumento de um ponto proporcional do SIVs promove o aumento de 2,31 pontos proporcionais do DC. Da mesma forma atuou o VDFVE, porém em menor extensão em pontos absolutos.

Sendo assim, temos que os animais dos grupos Polo e PSI respondem de forma muito semelhante quanto ao DC, e os animais QM de forma negativa. Isso quer dizer que, por exemplo, se tivermos um animal de cada grupo, com o mesmo tamanho de SIVs, o animal do grupo QM apresentará o menor valor de DC. E de forma igual será a resposta em relação ao VDFVE.

Para o VEj, os animais do grupo PSI influenciaram de forma negativa esta variável, e o grupo QM também, porém neste caso de forma menos significativa. 0 que chama a atenção neste caso é que os animais do grupo PSI apresentam nitidamente o maior VEj, e os maiores valores de dimensões cardíacas, não só neste trabalho, como em outros (Vöros 1991, Patteson 1995, Young 1999, Lightowler 2004) porém, para os animais aqui estudados, se selecionássemos um animal de cada grupo de mesmo tamanho, os animais do grupo PSI apresentariam o menor VEj - ou seja, entre animais com os mesmo valores de SIVs ou de VDFVE, independente da raça, os animais do grupo PSI apresentariam o menor valor de VEj. Neste ponto cabe a possibilidade de que, estes animais apresentam um bom rendimento cardíaco muito mais pelo seu tamanho, já definido pela própria seleção genética, do que por seu desenvolvimento em relação à atividade praticada.

Os animais do grupo Polo e QM apresentam melhor rendimento cardíaco, e este fato pode ser explicado pela adaptação ao treinamento físico desempenhado por estes, que neste caso se sobressai à influência exercida pela raça. Sendo assim, os animais que praticam atividades físicas de força conseguem atingir melhor desenvolvimento funcional cardíaco, do que quando exercido somente o treinamento de resistência.

\section{CONCLUSÕES}

0 treinamento físico misto, de força e resistência, permitiu o estabelecimento de hipertrofia excêntrica e concêntrica para os animais do grupo Polo e QM, tendo estes apresentado melhor resposta de função cardíaca em repouso.

Os animais do grupo PSI apresentaram notadamente corações maiores, porém, quando correlacionados com os outros grupos, apresentaram uma característica negativa sobre o volume de ejeção, sendo possível que o treinamento dos animais deste estudo não esteja explorando sua capacidade atlética máxima.

O septo interventricular apresenta influência importante sobre a resposta sistólica e diastólica do ventrículo esquerdo para todos os grupos de animais estudados, refletindo sobre as variáveis de função FEj e FS\%, que apresentaram alta correlação, sendo influenciadas diretamente pelo tamanho do septo interventricular, tanto em diástole como em sístole.

Os valores obtidos neste estudo podem auxiliar quando da avaliação de equinos atletas, podendo estes serem correlacionados com a atividade física desempenhada por estes animais.

Agradecimentos.- Ao Conselho Nacional de Desenvolvimento Científico e Tecnológico (CNPq) pelo suporte financeiro.

\section{REFERÊNCIAS}

Brunner M., Moeslinger T. \& Sieckermann P.G. 1995. Echocardiography for teaching cardiac physiology in practical student courses. Am. J. Physiol. 268:2-9.

Evans D.L. 1994. The cardiovascular system: anatomy, physiology, and adaptations to exercise and training, p.129-144. In: Hodgson D.R. \& Rose R.J. (Eds), The Athletic Horse: principles and practice of equine sports medicine. W.B. Saunders, Philadelphia.

Evans D.L. \& Rose R.J. 1988. Cardiovascular and respiratory responses to exercise in thoroughbred horses. J. Exp. Biol. 134:397-408.

Fagard R.H. 1997. Impact of different sports and training on cardiac structure and function. Cardiol. Clinics 15:397-412.

Laughlin M.H. 1999. Cardiovascular response to exercise. Adv. Physiol. Educ. 22:244-259.

Lightowler C.H., Piccione G., Giudice E., Del Olmo G.R. \& Cattáneo M.L. 2004. Echocardiography and electrocardiography as means to evaluate potential performance in horses. J. Vet. Sci. 5:259-262.

Long K.J. 1992. Two-dimensional and M-mode echocardiography. Equine Vet. Educ. 4:303-310.

Lyons A.S. \& Petrucelli R.J. 1987. Medicine: an illustrated history. Harry N. Abrams, New York. 603p.

Monganroth J., Maron B.J., Henry W.L. \& Epstein S.E. 1975. Comparative left ventricular dimensions in trained athletes. Annual Int. Med. 82:521-524.

Patteson M.W., Gibbs C., Wotton P.R. \& Cripps P.J. 1995. Echocardiographic measurements of cardiac dimensions and indices of cardiac function in normal adult Thoroughbred horses. Equine Vet. J. 19(Suppl.):18-27.

Pellicia A. \& Maron B.J. 1997. Outer limits of the athlete's heart: the effect of gender and relevance to the differential diagnosis with primary cardiac diseases. Cardiol. Clinics 15:381-396.

Poole D.C. \& Erickson H.H. 2008. Cardiovascular function and oxygen transport: responses to exercise and training, p.212-245. In: Hinchcliff K.W., Kaneps A.J. \& Geor R.J. (Eds), Equine Exercise Physiology. W.B. Saunders, Philadelphia.

Reef V.B. 1998. Cardiovascular ultrasonography, p.215-272. In: Ibid. (Ed.), Equine Diagnostic Ultrasound. W.B. Saunders, Philadelphia.

Vöros K., Holmes J.R. \& Gibbs C. 1991. Measurement of cardiac dimensions with two-dimensional echocardiography in the living horse. Equine Vet. J. 23:461-465

Young L.E. 1999. Cardiac responses to training in 2-year-old Thoroughbreds: an echocardiographic study. Equine Vet. J. 30(Suppl.):195-198.

Young L.E. 2003. Equine athletes, the equine athlete's heart and racing success. Exp. Physiol. 88:659-663.

Young L.E., Marlin D.J., Deaton C., Brown-Feltner H., Roberts C.A. \& Wood J.L. 2002. Heart size estimated by echocardiography correlates with maximal oxygen uptake. Equine Vet. J. 34(Suppl.):467-471. 